\title{
Características del Liderazgo Transformacional en Docentes de Lenguaje y Comunicación en una Muestra de Establecimientos Escolares de Vulnerabilidad Social
}

\author{
Characteristics of the transformational leadership of teachers of Language and Communi- \\ cation in a sample of educational settings in situation of social vulnerability.
}

\section{Características da liderança transformacional em docentes de linguagem e comuni- cação em uma mostra de estabelecimentos escolares de vulnerabilidade social}

\author{
Óscar Maureira $a^{\mathrm{a}}$ y Armando Rojas ${ }^{\mathrm{b}}$ \\ anstituto Interdisciplinario de Pedagogía y Educación, Universidad Católica Silva Henríquez. Correo elec- \\ trónico: omaureir@ucsh.cl. Telf.:(56-2) 27950522 \\ bUniversidad del Desarrollo. Correo electrónico: arojas@udd.cl. Telf.: (56-2) 2327934
}

\begin{abstract}
RESUMEN
El liderazgo colectivo, distribuido o compartido, emerge como propuesta para enriquecer, desde fuentes no tradicionales de influencias, el conocimiento, la formación y la mejora de la calidad de la intervención en los centros escolares. El presente estudio describe el liderazgo transformacional de los docentes de lenguaje y comunicación de $7^{\circ}$ básico a $4^{\circ}$ año medio en cinco establecimientos escolares que atienden a niños y jóvenes de escasos recursos. La descripción se basa en los resultados de la aplicación del cuestionario multifactorial de liderazgo a una muestra de 505 estudiantes. A través de parámetros de estadística descriptiva y el test de la prueba de t-student, se evidencia la inexistencia de diferencias estadísticamente significativas en la percepción del liderazgo del docente de lenguaje, según ciclos de enseñanza, como asimismo que los docentes de lenguaje son percibidos con rasgos transformacionales por sus estudiantes.
\end{abstract}

Palabras clave: liderazgo del docente, liderazgo transformacional, gestión del liderazgo en aula.

\begin{abstract}
Approaches such as collective leadership, distributed or shared, emerge - from non-conventional sources of influence - as proposals to enhance knowledge, education and the improvement of the teaching quality in educational settings in the area of leadership. This current study examines the transformational leadership of teachers of Language and Communication from 7 th grade of middle school education to 4th grade of secondary education in five educational institutions which work with deprived children and teenagers. The description is based on the results of a multifactorial questionnaire on leadership applied to a sample of 505 students. A lack of significant statistical differences in the perception of leadership of the teachers of Language and Communication, from both levels of education, is shown through descriptive statistics parameters and the T-student test. Similarly, these instructors are perceived as teachers with transformational features by their students.
\end{abstract}

Key words: leadership of teachers, transformational leadership, leadership management in the classroom.

\section{RESUMO}

Enfoques teóricos como a liderança coletiva, distribuída ou compartilhada, emergem como propostas para enriquecer, desde fontes não tradicionais de influências, o conhecimento, a formação e a melhora da qualidade da intervenção nos centros escolares no âmbito da liderança. Descreve-se a liderança transformacional dos docentes de linguagem e comunicação de $7^{\circ}$ série ao $4^{\circ}$ ano médio em cinco instituições escolares que atendem a crianças e jovens provenientes da classe baixa. A descrição se fundamenta nos resultados da aplicação do questionário multifatorial de liderança a uma mostra de 505 estudantes. Por meio de parâmetros de estatística descritiva e o teste da prova "t-student", evidenciou-se a inexistência de diferenças estatisticamente significativas na percepção da liderança do docente de linguagem, segundo 
os níveis de ensino, como também, que os docentes de linguagem são percebidos com propriedades transformacionais por seus alunos.

Palavras chave: liderança do docente, liderança transformacional, gestão da liderança em sala de aula.

\section{INTRODUCCIÓN}

La organización del sistema de la educación chilena atraviesa actualmente por un proceso de transformación. Así lo evidencia un conjunto de cambios y disposiciones tanto estructurales como legales- que han acaecido en el sistema escolar en los últimos cinco años. En este contexto, la tarea tanto de quienes desarrollan labores al interior de las aulas en los centros escolares, como de quienes conducen las escuelas, se convierte hoy por hoy en un desafío de máxima relevancia, especialmente considerando que la evidencia internacional (Volante, 2008) confirma el impacto conjunto de la variable efectividad docente y del liderazgo directivo en los resultados de aprendizaje de los alumnos. Esto conduce a pensar en la importancia que adquiere el factor liderazgo como proceso de influencia, tanto del directivo escolar como del docente de aula en la actualidad. Así, sobre la primera cuestión, distintos estudios recientes (Horn y Marfán, 2010; CEPPE, 2010; Reeves, 2010; Volante, 2010) y de política educativa en dirección escolar confirman la relevancia y pertinencia de fortalecer las capacidades de liderazgo pedagógico de los directores, como vía indirecta, pero consistente para superar la calidad de los aprendizajes en los estudiantes. No obstante, la segunda cuestión, es decir, sobre el nivel de conocimiento de capacidades de liderazgo de los docentes de aula y políticas de formación afines, parece no haber la misma magnitud de conocimiento e implementación.

Desde las tendencias sobre enfoques de liderazgo escolar y reestructuración de las organizaciones, se advierte, desde hace una década, una clara orientación hacia el estudio del liderazgo distribuido. Dicha concepción, con diferentes matices y controvertidas acepciones (Maureira, 2008) busca identificar al liderazgo más como una función de la organización que como un fenómeno personal individual y jerárquico. Así, la concepción de liderazgo colectivo, acuñada por Leithwood y Mascall (2008) precisa fuentes de liderazgos, tales como: apoderados, estudiantes, coordinadores de sectores de aprendizajes, docentes de aula que representarían, en una estructura de alineamiento planificado, una fuerza de influencia determinante en la mejora de la calidad de los aprendizajes, tanto organizacionales como estudiantiles.

Dentro de la diversidad de facetas esenciales de desempeño de los docentes de aula, entre otras, está la de implicar y motivar a los estudiantes, a través de un repertorio de recursos, hacia la mejora de sus aprendizajes. Si bien las capacidades -en el saber pedagógico y disciplinar- son fundamentales, también lo son, de modo quizá determinante para evaluar la total efectividad del docente, aquellas de relación interpersonal con sus estudiantes. En este contexto, cobra sentido el conocimiento sobre las capacidades de liderazgo que el docente pueda evidenciar, a fin de contrastar con las percepciones que los estudiantes tienen de las capacidades de sus docentes. De este modo, el siguiente trabajo, con características de exploratorio y descriptivo, busca caracterizar el liderazgo del docente de aula del sector de aprendizaje Lenguaje y Comunicación. Para estos fines se precisa en primer lugar, una breve sistematización del conocimiento en torno al liderazgo tanto directivo como docente, para luego precisar interrogantes, objetivos, diseño, resultados y conclusiones del estudio. 


\section{LIDERAZGO DIRECTIVO ESCOLAR: UNA BREVE CARACTERIZACIÓN}

Los estudios y reflexiones de Hargreaves (2006), Leithwood (2009), Hopkins (2009), Fullan (2007), Robinson (2010), Elmore (2010), Bolívar y Maureira (2010) y MacBeath (2011) dejan de manifiesto el liderazgo educativo como factor estratégico para la mejora educativa. En este sentido, se identifica la centralidad del liderazgo como cualidad organizativa, a diferentes niveles, para fines de innovación y mejoramiento escolar. Varios estudios dan cuenta de los efectos y peso específico que tiene este componente en la organización escolar. Éstos presentan diversos enfoques para conceptualizar y operacionalizar el liderazgo educacional (Hallinger y Murphy, 1985; Leithwood, 1994; Sergiovanni, 1984; Volante, 2002; Carbone, 2006, entre otros). Así, los primeros estudios internacionales de eficacia escolar (Edmonds, 1983; Purkey y Smith, 1985; Reynolds et al., 1997) destacan al liderazgo pedagógico del director como una variable consistente en la red de factores asociados a desempeños notables de establecimientos educativos de enseñanza primaria y secundaria. Posteriormente, con la profundización y refinamiento de métodos y técnicas de análisis de datos, orientadas a contrastar modelos de eficacia escolar integrados (Scherenes y Creemers, 1996), y evaluar qué tipo de influencia se adecua más a las exigencias de los establecimientos escolares, surgen enfoques más centrados en la organización y coordinación de acciones al interior del establecimiento. Se destacan en especial aspectos asociados a la construcción de misión y visión del establecimiento educativo. De este modo, enfoques como el liderazgo transformacional de B. Bass y Avolio (1994) y el liderazgo transformacional escolar de Leitwhood (1994, 2009) nos sitúan de manera más concreta en el concepto de liderazgo y sus efectos en la mejora y reestructuración escolar. Asimismo, los Informe McKinsey (Barber y Moursshed, 2008), OCDE (Ponts et al., 2008) y el National College of School Leadership (NCSL, 2006) señalan que, más allá de las diferencias culturales y de idiosincrasia, el factor liderazgo constituye el segundo factor de explicación de la calidad de los aprendizajes en los establecimientos educativos. En esta línea de formación en capacidades y en desarrollo de la organización, el Informe Internacional TALIS (2009) comparó las condiciones de enseñanza y aprendizaje de 23 países integrantes de OCDE y dio cuenta que en el ámbito de la dirección educativa:

Existen muchas posibilidades de que los profesores aprendan de sus colegas, aunque los profesores comunican una colaboración relativamente infrecuente entre el conjunto del profesorado, excepto en lo que se refiere a un mero intercambio de información. La mejora de esta situación requerirá una adecuada dirección educativa, así como el desarrollo de políticas eficaces de desarrollo de recursos humanos en los centros (Informe TALIS, 2010: 25).

Con respecto a la investigación en Chile, Horn y Marfán (2010) señalan que en cinco trabajos basados en eficacia escolar se identifican tres pilares del liderazgo: a) Pilar técnico, que se relaciona con la experticia pedagógica del director. Forma parte de este pilar lo que Belleiet et al. (Cit. en Horn y Marfán, 2010) señalan como saberes, permitiendo al director ganar respeto de la comunidad, dar apoyo técnico a los docentes, evaluar sus prácticas, planificar los procesos educativos y monitorear los aprendizajes; b) Pilar de manejo emocional y situacional, que implica ser capaz de conducir la relación que se establece con los docentes. Elementos relacionados son la capacidad para motivar, para trabajar en equipo, velar por un buen clima escolar, demostrar altas expectativas en alumnos y docentes, escuchar y abrir espacios de participación; c) Pilar organizacional, 
siendo propio de un buen líder la capacidad para estructurar una organización escolar eficiente, esto es, facilitar las condiciones para que los docentes puedan realizar un buen trabajo (Cit. en Horn y Marfán, 2010). Estos resultados son consistentes con los resultados del Estudio de Casos de Eyzaguirre y Fontaine (2008) sobre las Escuelas que tenemos. Todo esto contrasta con los resultados de la encuesta de opinión que hizo el Centro de Políticas Sociales de la Universidad Central, sobre las atribuciones de responsabilidades de los directores en los logros del SIMCE, quienes reconocen en un $4 \%$ sus compromisos profesionales sobre este importante indicador de logro. Cuestión que se observa también en lo clave que resulta en los Programas de Asistencia Técnica (ATE) y sus efectos, que el equipo directivo posea un alto liderazgo académico, reconocido y a toda prueba, ya que como advierte Bellei et al. (2010), en la síntesis de las dimensiones de efectividad de dichas ATE, debe haber una capacidad de liderazgo importante para que estos Programas de Mejoramiento Educativo tengan el efecto que se espera de ellos. Asimismo, Vegas (2010), a raíz de un estudio sobre políticas comparadas de formación docente, sostiene sobre el rol de los directores que "es necesario sean líderes académicos y que puedan apoyar al docente, las investigaciones confirman cada vez más el importante rol que juegan en el aprendizaje. Un buen director sabe quiénes son sus mejores maestros y quiénes son los que necesitan más apoyo". En consecuencia con lo anterior, este estudio centra su objetivo en el liderazgo del docente.

\section{LIDERAZGOS PARA EL APRENDIZAJE}

El enfoque del liderazgo para el aprendizaje (Bolívar, 2010) sostiene que el objetivo prioritario de las políticas educacionales en el siglo XXI debiera ser garantizar a todos los estudiantes los aprendizajes imprescindibles que les que posibiliten, sin riesgo de exclusión, la integración y participación activa en la vida pública. El liderazgo en la enseñanza está, sin duda, para hacerlo posible. Asimismo, señala que se requiere de una formación inicial y en servicio adecuadas. Al hacer una revisión por las mallas curriculares de las carreras de pedagogía tanto en las universidades como en los institutos profesionales del país, se observa que no son incluidas ejes o áreas de formación que desarrollen habilidades de liderazgo. No obstante, desde diversos estudios, políticas y enfoque evaluativos del docente, se infiere que dicha capacidad (liderazgo) se requiere, cada vez más, en la práctica escolar y pedagógica. El profesorado, según el Programa Golden 5 (Lera et al., 2007) tiene las opciones de estratega o comunicante para ejercer su liderazgo en el aula. La primera tiene como foco de acción prioritaria el apego al cumplimiento de la planificación curricular. En cambio, la segunda, se focaliza prioritariamente en la construcción de diálogo con los estudiantes, a objeto de comprenderlos para motivarlos e implicarlos en la tarea de aprender. Sin lugar a duda, como dice la misma referencia, subyace a esta última dimensión un tipo de interacción de influencia formativa que privilegia la relación con los alumnos y entre los alumnos.

Dentro del contexto reformista que se viene implementando casi década a década en los sistemas educativos, algunos autores (Alvarado y Coyle, 1997) sostienen que es fundamental desarrollar el rol de profesor (maestro) líder, en base a estrategias de poder de abajo hacia arriba de involucramiento en el mejoramiento escolar. Las funciones más características de tal rol corresponderían, entre otras, a generar dispositivos para compartir 
conocimiento sobre la práctica pedagógica, difundir principales hallazgos sobre la enseñanza y el aprendizaje, implementar proyectos de investigación-acción, participar con los demás actores de la comunidad educativa, ser mentor de profesores noveles. Pero dichas funciones, para ser efectivas, deben ser apoyadas por los directores, ya que a menudo quedan fuera de los circuitos de decisión escolar y, por otro, cuando se le dan tareas de liderazgo, carecen de habilidades de influencia que les permitan mayores atribuciones.

Desde una perspectiva más cultural, política y no funcional, Coronel (2005) describe y tensiona la perspectiva del liderazgo del profesorado desde una óptica de construcción social y de significados. Con lo cual el liderazgo del profesorado es conceptualizado como "una forma de mediación que implica sobre todo una redistribución del poder y la influencia dentro de la escuela como organización". (Coronel, 2005: 474). Así, en primer lugar, precisa dicho autor que el campo de investigación sobre el liderazgo del profesorado es incipiente, debido a que gran parte de la producción de conocimiento sobre liderazgo ha estado en la dirección escolar, tratándose en gran parte su conocimiento, desde una perspectiva funcionalista y con base a un fenómeno individual, heroico y jerárquico. En segundo lugar, las políticas educativas contemporáneas han acentuado la descentralización y autonomía de los centros educativos en la toma de decisiones para la mejora e innovación. A partir esto y en un contexto de mayor democratización en los centros escolares, es posible esperar mayor implicación e influencia de los profesores, es decir, liderazgos de estos para intervenir en los ámbitos sobre el profesorado (práctica de la clase, capacidad personal e interpersonal), sobre la escuela como organización (estructuras y procesos, cultura y capacidad), más allá de la escuela (crítica y debate, creación y transferencia de conocimiento, mejora en el capital social de la comunidad), sobre los aprendizajes de los estudiantes (atención, disposición y metacognición). Finalmente, dicho autor, en base a la construcción de un discurso positivo y esperanzador sobre el liderazgo del profesorado, plantea que el tema se presenta como una oportunidad para analizar e indagar sobre la situación del profesor en la escuela, la promoción de la toma de decisión compartidas y el trabajo entre colegas, las interrelaciones entre la dirección y el profesorado, el desarrollo profesional del colectivo docente y la propia organización escolar.

Por otro lado, desde una perspectiva didáctica, Ramos (2005) ha acuñado el concepto de liderazgo didáctico. Tal acepción se origina por la complementariedad de la definición de liderazgo de Tannenbaum et al. (1961) y el de didáctica, que define como "el estudio discursivo, colaborativo y crítico de la interacción en el salón de clases entre el(la) maestro(a), los estudiantes y el currículo con el propósito de lograr un mundo justo y democrático" (Ramos, 2005: 1). Desde esta perspectiva, los ámbitos de intervención educativa en que se vería reflejado tal liderazgo didáctico estarían en crear una cultura de la paz en la escuela, dirigida a construir comunidades de aprendizaje y climas organizacionales positivos, desarrollar programas de enseñanza que redunde en aprendizajes de los estudiantes y aplicar las mejores prácticas para lograr los mejores aprendizajes en los estudiantes.

Con respecto a este último punto, es decir, las variables y factores asociados al aprendizaje escolar existen, en términos generales, tres grandes tradiciones que han abordado esta temática (Cornejo y Redondo, 2007). En primer lugar, es posible identificar la tradición de estudios de los procesos de enseñanza y aprendizaje escolares basados en las teorías del aprendizaje por reestructuración (Coll et al., 2001). En esta tradición, por cierto, muy heterogénea, el foco está puesto en aquellos procesos de aprendizaje relevantes 
desde las llamadas teorías constructivistas del aprendizaje humano y su ocurrencia en el contexto de la sala de clases (significatividad de los aprendizajes, procesos de mediación, actividad mental de los estudiantes, sentido y pertinencia social de los aprendizajes). No existe en esta tradición un afán comparativo ni una mirada jerarquizadora respecto de la importancia de estos factores asociados. En segundo lugar, se puede mencionar la tradición de estudios que intentan comprender la dinámica de la escuela como una institución compleja y los procesos de aprendizaje que ocurren en su interior. No se trata en este caso de una única escuela de pensamiento, sino de corrientes de investigación que comparten una mirada de causalidad no lineal de los procesos y resultados en la escuela, así como una visión no racionalista sino cultural de las instituciones escolares. Destacamos en este sentido los estudios de la mejora escolar (Hopkins y Lagerweij, 1997; Bolívar, 2000) y los estudios centrados en los procesos culturales de la escuela (Fullan, 1993; Hargreaves, 1994). Por último, es posible mencionar la tradición de estudios sobre eficacia escolar con sus distintos énfasis y áreas de investigación (Murillo, 2003).

Todos los Informes, tanto nacionales como internacionales, sobre efectividad escolar y logros de aprendizaje en estudiantes, destacan el papel determinante de la calidad del docente como el primer factor de explicación de la calidad de los aprendizajes. Si bien en este ámbito se han elaborados importantes marcos para orientar a los profesores en la mejora de sus prácticas formativas, pocos han sido las aproximaciones investigativas de las competencias de liderazgo y sus efectos en variables tales como: disposición para el aprendizaje, colaboración entre estudiantes para el aprendizaje, entre otros. No obstante, Figueroa y García (2010) describen un conjunto de investigaciones y reflexiones sobre la necesidad de un nuevo liderazgo. En particular el trabajo de Jiménez et al. (2010) releva lo clave que es el liderazgo de los profesores en la contribución a los logros de los estudiantes, insistiendo en ámbito asociados a dicha competencia: al respecto, se señala que:

"Para que el profesor sea un líder dentro del aula, éste debe estar reconocido por sus alumnos como tal. La autoridad del líder docente proviene siempre de los estudiantes con los cuales interactúa y con quienes comparte su posición, normas y valores. Un profesor líder se caracteriza por su ascendencia y autoridad moral sobre los estudiantes, la cual obedece a un proceso de influencia construida por el ejemplo y las buenas prácticas del docente" (Jiménez, Figueroa y García, 2010: 80).

Dentro de las características que comportan las competencias de liderazgo del profesor, Jiménez et al. (2010) las contextualiza en las dimensiones de: facilitación del aprendizaje, agente de cambio, comunicador y motivador coherente, los rasgos de un docente que pretende ser reconocido, por sus estudiantes, como líder. En el mismo sentido, el trabajo de Uribe (2005) profundiza sobre liderazgo docente, identificando 12 de 21 responsabilidades asociadas al profesor como líder. El siguiente cuadro retrata dichas responsabilidades y manifestaciones.

Cuadro 1. Responsabilidades del liderazgo docente (Uribe, 2005).

\begin{tabular}{|c|c|}
\hline $\begin{array}{c}\text { Ámbitos de } \\
\text { responsabilidad }\end{array}$ & Descripción \\
\hline 1. Cultura & $\begin{array}{r}\text { El profesor y todos los miembros de la comunidad educativa comparten } \\
\text { creencias, sentido de comunidad y cooperación. }\end{array}$ \\
\hline
\end{tabular}




\begin{tabular}{|c|c|}
\hline 2. Orden & $\begin{array}{l}\text { El profesor tiene establecido los procedimientos y rutinas de sus clases, en } \\
\text { otras palabras, planifica sus clases. }\end{array}$ \\
\hline 3. Disciplina & $\begin{array}{l}\text { El profesor procura que los estudiantes no pierdan el foco hacia distractores y } \\
\text { mantengan un clima adecuado para el aprendizaje. }\end{array}$ \\
\hline 4. Recursos & $\begin{array}{c}\text { El profesor entrega y usa de manera adecuada y pertinente los materiales y } \\
\text { recursos a sus estudiantes. }\end{array}$ \\
\hline \multirow[b]{2}{*}{ 5. Enfoque } & El profesor establece objetivos claros y alcanzables para sus estudiantes. \\
\hline & $\begin{array}{l}\text { El profesor posee amplio conocimiento del currículo, estrategias y métodos } \\
\text { de enseñanza y evaluación. }\end{array}$ \\
\hline $\begin{array}{l}\text { 7. Estímulo en } \\
\text { lo cotidiano } \\
\text { (recompensa) }\end{array}$ & El profesor reconoce y premia los logros de sus estudiantes. \\
\hline 8. Comunicación & $\begin{array}{l}\text { El profesor mantiene una fluida y continua comunicación con sus estudiantes, } \\
\text { pares, director y apoderados. }\end{array}$ \\
\hline 9. Incorpora & $\begin{array}{l}\text { El profesor involucra y compromete a los apoderados en el aprendizaje de los } \\
\text { estudiantes. }\end{array}$ \\
\hline $\begin{array}{l}\text { 10. Agente de } \\
\text { Cambio }\end{array}$ & $\begin{array}{c}\text { El profesor tiene la voluntad y lidera el cambio y la mejora continua, } \\
\text { desafiando activamente el status quo. }\end{array}$ \\
\hline 11. Optimizador & El profesor inspira y lidera nuevas y desafiantes innovaciones. \\
\hline $\begin{array}{l}\text { 12. Ideales/ } \\
\text { Creencias }\end{array}$ & $\begin{array}{l}\text { El profesor comunica y opera desde fuertes ideales y creencias sobre la } \\
\text { educación. }\end{array}$ \\
\hline
\end{tabular}

Desde la perspectiva de la lógica de los estándares, en Jiménez et al. (2010), la Comisión de Estándares Profesionales para la Enseñanza del Estado de Carolina del Norte en los Estados Unidos de América (2007) establece los siguientes estándares para el ejercicio de la profesión docente en las escuelas del Estado:

Cuadro 2. Estándares Profesionales para la Enseñanza (Jiménez et al., 2010).

\begin{tabular}{|c|c|}
\hline Estándares & Manifestaciones \\
\hline 1 & Los profesores demuestran liderazgo. Ellos guían en sus salas de clases. \\
\hline 2 & $\begin{array}{c}\text { Los profesores establecen un ambiente respetuoso para poblaciones diversas } \\
\text { de estudiantes }\end{array}$ \\
\hline 3 & Los profesores conocen los contenidos que enseñan \\
\hline 4 & Los profesores facilitan el aprendizaje de sus estudiantes \\
\hline 5 & Los profesores se reflejan en sus prácticas. \\
\hline
\end{tabular}

Sin duda lugar a dudas, cuando la prioridad es la mejora sustantiva de la calidad de los aprendizaje de los estudiantes, las capacidades de influencia no solo basadas en el poder conferido por un título de profesor, es decir, liderazgo, hacen la diferencia en la agregación de valor a la tarea formativa, reconocida por los estudiantes y el mismo 
docente. En síntesis, es posible afirmar que el liderazgo en aula, es decir, la capacidad del docente para influir en mayores y mejores aprendizajes, aparece como un foco de conocimiento relevante para dimensionar las características de efectividad docente. Desde esta perspectiva y adhiriendo al enfoque de Bass y Avolio (2000), el profesor puede ejercer su liderazgo en una perspectiva más transaccional (recompensar de diversas maneras) o transformacional (cambio significativo en el aprender) o dejar hacer (cumplir administrativamente con el rol de instructor).

\section{PROBLEMA, OBJETIVOS Y COMPONENTES DEL DISEÑO DE INVESTIGACIÓN}

Por lo anterior, resulta pertinente preguntarse: ¿Es posible identificar factores de liderazgo docente al interior del aula? ¿Cuál es la situación del liderazgo transformacional de los docentes chilenos? Esta situación se torna aún más relevante para el caso de los docentes que trabajan en sectores de alto riesgo, pues en tal escenario su papel es aún más decisivo para la formación de decenas de miles de jóvenes en situación de vulnerabilidad. En este sentido, resulta interesante acercarse a instituciones educacionales o fundaciones sin fines de lucro que trabajan en estos sectores y que han tenido buenos resultados, ya que podría pensarse que una de las variables que en mayor medida inciden en la obtención de buenos resultados es la variable profesor. Especialmente, aquellos que interactúan más tiempo con sus estudiantes y que están en sectores de aprendizaje vitales para el desarrollo de otras. Por las diversas argumentaciones dadas anteriormente, resulta asimismo interesante preguntarse: ¿Cómo es el liderazgo transformacional de los docentes de lenguaje y comunicación en los establecimientos escolares de vulnerabilidad social? ¿Existen diferencias significativas entre la percepción sobre el liderazgo del docentede lenguaje en los alumnos de educación básica con respecto a la percepción de los alumnos de educación media? De este modo, el propósito de la investigación es describir el liderazgo transformacional de los docentes de lenguaje y comunicación de $7^{\circ}$ año básico a $4^{\circ}$ año medio de cinco establecimientos escolares que atienden a niños y jóvenes provenientes de sectores vulnerables, considerando la percepción de los estudiantes. La presente investigación correspondió a un diseño de tipo no experimental transversal. El criterio de muestreo de este estudio fue de muestreo intencional; se prefirió este tipo de muestra por tratarse de un estudio exploratorio, cuyo principal objetivo consistió solo en realizar un primer acercamiento al fenómeno en estudio ( Hernández, Fernández y Baptista, 2003). En particular, este se configuró sobre una población compuesta por todos los profesores de Lenguaje y Comunicación de $7^{\mathrm{a}}$ año básico a $4^{\mathrm{a}}$ año medio en cinco colegios pertenecientes a una Fundación que por misión atiende a niños y jóvenes en riesgo social. Se aplicaron cuestionarios a 15 profesores de Lenguaje y Comunicación y a 505 estudiantes en 5 colegios durante los meses de abril, mayo y junio del año 2010. Esto corresponde al $62,5 \%$ del total de 8 establecimientos que forman parte de dicha Fundación, mientras que los 15 docentes y los 505 estudiantes (es decir, 15 cursos) corresponden al 63\% del total de 24 profesores de este subsector (Lenguaje) de toda la Fundación. El instrumento que se utilizó para llevar a cabo el presente estudio corresponde a una adaptación del Cuestionario Multifactorial sobre Liderazgo (Bass y Avolio, 2000). Ahora bien, para este estudio en particular se tomó solo la dimensión transformacional, 
puesto que es la variable que mejor explica la varianza de todo el modelo (Guajardo Luna et al., 2004). Para analizar los resultados, se llevaron a cabo dos tipos de análisis. En primer lugar, se realizó un análisis descriptivo y a continuación se aplicó una prueba t de student. Con respecto al análisis descriptivo se tomaron las cinco dimensiones del MLQ asociadas al Liderazgo Transformacional. En este punto es importante aclarar que para categorizar los resultados se han establecido distintos niveles de liderazgo. Estos niveles son el resultado de la suma de los porcentajes correspondientes a las categorías bastante a menudo y siempre.

Tabla 1. Categorización de los niveles de liderazgo obtenidos por los docentes

\begin{tabular}{|c|c|}
\hline CATEGORÍA & PORCENTAJE \\
\hline Tendencia muy alta liderazgo & $100 \%$ a $80 \%$ sumatoria de opción siempre y bastante a menudo \\
\hline Tendencia alta liderazgo & $79 \%$ a $60 \%$ sumatoria de opción siempre y bastante a menudo \\
\hline Tendencia media liderazgo & $59 \%$ a $40 \%$ sumatoria de opción siempre y bastante a menudo \\
\hline Tendencia baja liderazgo & $39 \%$ a $20 \%$ sumatoria de opción siempre y bastante a menudo \\
\hline Tendencia muy baja liderazgo & $19 \%$ a $00 \%$ sumatoria de opción siempre y bastante a menudo \\
\hline
\end{tabular}

En segundo lugar, se aplicó una prueba $t$ de student ${ }^{1}$ para determinar la existencia de diferencias significativas entre la percepción sobre el liderazgo del docente de lenguaje en los alumnos de educación básica (en este caso $7^{\circ}$ y $8^{\circ}$ ) con respecto a la percepción de losalumnos de educación media (para este caso de $1^{\circ}$ a $4^{\circ}$ medio). El propósito de ello fue establecer un pie para encontrar cuáles son algunas de las características más valoradas con respecto al liderazgo de sus docentes por los alumnos de básica en relación con los estudiantes de media. En concordancia con lo anterior, para el presente estudio se plantea la siguiente hipótesis general de trabajo: No existen diferencias significativas entre la percepción sobre el liderazgo del docente de lenguaje y comunicación en los estudiantes de educación básica con respecto a la percepción de los estudiantes de educación media al considerar las cinco dimensiones del liderazgo transformacional. Ahora bien, para efectos de este estudio, esta hipótesis general ha sido desagregada en 5 hipótesis parciales que siguen la misma formulación, pero enunciadas por la dimensión respectiva.

\section{PRINCIPALES RESULTADOS, CONCLUSIONES Y REFLEXIONES}

La siguiente tabla resume los resultados obtenido en los cinco colegios en cada una de las dimensiones transformacionales, por categoría.

1 La prueba t de student es un estadístico de contraste utilizado para probar la hipótesis nula planteada (las medias de los dos grupos son iguales) y se construye en función de las diferencias registradas entre los valores de la variable de estudio evaluada en cada uno de los grupos a comparar. Para ello se utiliza la información procedente de las medias y desviaciones estándar de cada uno de los grupos de estudio. Para poder aplicar esta prueba es necesario determinar si las varianzas de ambos grupos son iguales o no. Para ello se aplica el Test de Levene, que entrega esta información. 
Tabla 2. Porcentaje obtenido en promedio por los docentes de los cinco colegios, por dimensión.

\begin{tabular}{|c|c|c|c|c|}
\hline Dimensión & Siempre & $\begin{array}{c}\text { Bastante a } \\
\text { menudo }\end{array}$ & $\begin{array}{c}\text { Algunas } \\
\text { veces/raras } \\
\text { veces/ nunca }\end{array}$ & Total \\
\hline Influencia idealizada como atributo & $64.62 \%$ & $33,42 \%$ & $1,96 \%$ & $100 \%$ \\
\hline $\begin{array}{c}\text { Influencia idealizada como } \\
\text { comportamiento }\end{array}$ & $62,72 \%$ & $9,5 \%$ & $27,78 \%$ & $100 \%$ \\
\hline Motivación inspiracional & $31,14 \%$ & $31,30 \%$ & $37,56 \%$ & $100 \%$ \\
\hline Estimulación intelectual & $61,74 \%$ & $13,32 \%$ & $24,94 \%$ & $100 \%$ \\
\hline Consideración individualizada & $43,6 \%$ & $3,70 \%$ & $52,7 \%$ & $100 \%$ \\
\hline
\end{tabular}

Con respecto a los resultados de esta investigación se puede afirmar que la dimensión influencia idealizada como atributo es la dimensión del liderazgo transformacional que obtiene el mayor porcentaje, con un $98,04 \%$, considerando las opciones siempre y bastante a menudo. En relación a la dimensión influencia idealizada como comportamiento, esta dimensión obtiene un $72,22 \%$. Esto permite desprender -en comparación con los resultados de la dimensión anterior- que, si bien los estudiantes de $7^{\circ}$ básico a $4^{\circ}$ medio perciben en sus profesores atributos de liderazgo, no siempre los observan en su comportamiento. Esto conduce a pensar en que, tal vez, esta percepción cambiaría en la medida en que los equipos directivos de los colegios permitan a sus docentes desarrollar sus atributos de liderazgo, en otras esferas que no necesariamente debieran ser las de la sala de clases. Con respecto a la dimensión motivación con inspiración, esta dimensión obtiene un $62,44 \%$. El porcentaje obtenido es relativamente alto, pero comparativamente más bajo que las otras dimensiones. Este resultado permite sentar las bases para futuras investigaciones que relacionen en forma más precisa la motivación y el liderazgo, pues allí pueden aparecer datos que contribuyan al mejoramiento del clima al interior del aula. En relación a la dimensión estimulación intelectual se puede señalar que esta dimensión obtiene un 75,06\%. En términos generales, esta dimensión es la que obtiene el segundo porcentaje más alto, lo que resulta bastante interesante, por cuanto estos resultados constituyen evidencia concreta que permitiría direccionar la política educativa y curricular. Estos resultados permiten pensar que en la medida que los profesores puedan desarrollar actividades al interior de la sala que estimulen intelectualmente a sus estudiantes, es posible obtener mejores aprendizajes. Por último, con respecto a la dimensión consideración individualizada se puede afirmar que esta dimensión obtiene un 46,76\%. Este resultado es el más bajo de todas las dimensiones analizadas. Ello puede tener su explicación en el alto número de alumnos por cada curso, lo que no permite una adecuada dedicación del profesor con cada uno de sus estudiantes. Esto sugiere que en la medida que tengamos a docentes líderes y con salas de clases con cursos menos numerosos, podrían mejorar los aprendizajes de los estudiantes.

En relación a la aplicación de la prueba $t$ de student. Los resultados obtenidos permitieron aceptar la hipótesis nula. Es decir, no hay diferencias significativas entre la percepción sobre el liderazgo del docente de Lenguaje y Comunicación en los estudiantes 
de educación básica con respecto a la percepción de los estudiantes de educación media considerando el contraste para cada una de las dimensiones transformacionales.

Este estudio aporta evidencia, aunque bastante incipiente debido a su diseño, sobre la influencia que tiene el liderazgo del docente sobre los resultados de aprendizaje. En esta misma línea de análisis, los resultados arrojados en esta investigación vienen a complementar la hipótesis de Hallinger y Heck (Cit. en Volante, 2008) y de Seashore-Louis et al. (Cit. en Bolívar-Botía, 2010), quienes identifican la instrucción en el aula como la variable que mayor impacto tiene sobre el aprendizaje de los estudiantes. Todo lo anterior conduce a pensar que, en la medida que se tengan profesores considerados como líderes transformacionales por sus estudiantes, sería posible avanzar en la explicación de la efectividad docente, expresadas en resultados de aprendizaje. Por lo anterior, estos resultados abren un camino para futuras investigaciones y constituye una interesante línea de producción de conocimiento, asociada al liderazgo distribuido. Con respecto a las diferencias entre la percepción sobre el liderazgo del docente de lenguaje en los alumnos de educación básica con respecto a la percepción de los alumnos de educación media, los resultados evidencias cierta constante. Es decir, que los patrones de influencia son más estables que diferenciados. Finalmente, los resultados de este estudio, exploratorio y descriptivo, invitan a generar investigaciones de mayor alcance a objeto de delinear potencialidades y evaluar capacidades de liderazgo del docente aula para la innovación y la mejora de los aprendizajes de los estudiantes.

\section{REFERENCIAS BIBLIOGRÁFICAS}

Alvarado, C. (1997). If leadership was everyone's domain.Inm taking the lead: Investing in early childhood leadership for the 21st century. Boston, MA: WheelockCollege.

Anderson, S. (2010). Liderazgo directivo: claves para una mejor escuela. Revista Psicoperspectivas, vol. 9, n. 2, 34-52.

Ahumada., L. (2010). Liderazgo distribuido y aprendizaje organizacional: Tensiones y contradicciones de la ley de subvención escolar preferencial en un contexto rural. Psicoperspectivas, vol. 9, n. 1, 111-123.

Barber, M. y Mourshed, M. (2007). How the World's Best-Performing School Systems Come Out On Top. London: McKinsey \& Company.

Belleï, C. (2004). Escuelas eficaces en sectores de pobreza. Santiago: UNICEF.

Bolívar-Botía, A. (2010). El liderazgo educativo y su papel en la mejora: una revisión actual de sus posibilidades y limitaciones. Revista Psicoperspectivas, vol. 9. n. 2, 9-33.

Bolívar-Botía, A. (2010). ¿Cómo un liderazgo pedagógico y distribuido mejora los logros académicos? Revisión de la investigación y propuesta. Revista Internacional de Investigación en Educación, vol. 3, n. 5, 79-106.

Carbone, R. (2008). Situación de liderazgo educativo en Chile. Santiago: Ministerio de Educación, Universidad Alberto Hurtado.

CEPPE (2009). Prácticas de Liderazgo Directivo y Resultados de Aprendizaje. Hacia Conceptos Capaces de Guiar la Investigación Empírica. REICE. Revista Iberoamericana sobre Calidad, Eficacia y Cambio en Educación, vol. 7, n. 3, 19-33.

Cornejo, R. y Redondo, J. M. (2007). Variables y factores asociados al aprendizaje escolar: una discusión desde la investigación actual. Revista Estudios Pedagógicos, vol. 33, n. 2, 155-175.

Coronel, J. M. (2005). El liderazgo del profesorado en las organizaciones educativas: temáticas para su análisis e investigación. Revista Española de Pedagogía, n. 232, 471-490 
Coyle, M. (1997). Teacher leadership vs. school management: Flatten the hierarchies. ClearingHouse, vol. 70, n. $5,236-239$.

Edmonds, R. (1983). Search for effective school: the identification and analysis of city school that are instructionally effective for poor children. Michigan: East Lansing.

Elmore, R. (2010). Mejorando la escuela desde la sala de clases. Santiago: Fundación Chile- CAP.

Figueroa, C. y García M., M. (Ed.). (2010). Nuevos Tiempos, Nuevas Necesidades. Importancia de un Liderazgo Diferente para las Comunidades Escolares. Valparaíso: Ediciones Universidad Playa Ancha.

Fullan, M. (2007). Leading in a Culture of Change. San Francisco, CA: Jossey-Bas.

Garay, S y Uribe, M (2006). Dirección Escolar como factor de eficacia y cambio. Situación de la Dirección Escolar en Chile. Revista Electrónica sobre Calidad, Eficacia y Cambio Escolar, vol. 4, 39-64.

Hallinger, P. \&Murphy, J. (1985). Assessing the Instructional Management Behavior theof principal. Elementary School Journal, n. 86, 212-247.

Hargreaves, A. \&Godson, I.(2006). Educational Change Over Time? The Sustainability and Nonsustainability of Three Decades of Secondary School Change and Continuity. EducationalAdministrationQuarterly, vol. 4, n. 1, 3-41.

Hargreaves, A. y Fink, D. (2006). El Liderazgo Sostenible. Siete Principios para el Liderazgo en Centros Educativos Innovadores. Madrid: MINEDUC / EdicionesMorata.

Hopkins, D. (2000). Instructional Leadership and School Improvement.National En línea, disponible en: http://mt.educarchile.cl/mt/jjbrunner/archives/Instructional_leadership.pdf (15/05/2012).

Horn, A. \& Marfán, J. (2010). Relación entre liderazgo educativo y desempeño escolar: revisión de la investigación en Chile. Revista Psicoperspectivas, vol. 9, n. 2, 82-104.

Leithwood, K. (1994). Leadership for School Restructuring. Educational Administration Quarterly, vol. 30, n. 4, 498-518.

Leithwood, K y Mascall, B. ( 2008). The effects of collective leadership on studen achievement, Educational Administration Quartely, vol. 44, n. 4, 529-561.

Leithwood, K. (2009). ¿Cómo liderar nuestras escuelas, Aportes desde la investigacion? Área de Educación. Fundación Chile. Santiago: Salesianos.

López, V. (2010). Liderazgo y mejora educativa. Revista Psicoperspectivas, vol. 9, n. 2, 1-8.

Macbeath, J. (2011). Liderar el aprendizaje dentro y fuera de la escuela. Área de Educación. Fundación Chile. Santiago: Salesianos.

Maureira, O. (2004). El Liderazgo factor de eficacia escolar. Hacia un modelo causal. Revista Electrónica sobre Calidad, Eficacia y Cambio Escolar, vol. 2, n. 1. En línea; disponible en: http://www.ice.deusto.es/RINACE/reice/vol2n1/Maureira.pdf (15/05/2012).

Maureira, O. (2006). Dirección y eficacia escolar, una relación fundamental. Revista Electrónica sobre Calidad, Eficacia y Cambio Escolar, vol. 4., n. 4. En línea; disponible en: http://www. rinace.net/arts/vol4num4e/art1.pdf (15/05/2012).

Maureira, O. (2008). Liderazgo para la eficacia escolar: una estrategia para la calidad. Santiago: Ediciones Universidad Cardenal Silva Henríquez.

Maureira, O. (2008). Tendencias del liderazgo directivo. Boletín de Investigación, vol. 23, n. 2, 81-102.

Molinar, M y Velázquez, L. (2005). Liderazgo en la Labor Docente. Trillas: México.

Murillo, J. (2003). El movimiento de investigación de Eficacia Escolar. En J. Murillo (Coord.), La investigación sobre Eficacia Escolar en Iberoamérica. Revisión internacional del estado del arte (pp. 53-92). Bogotá: Convenio Andrés Bello.

National College of School Leadership (2006). Seven Strong Claims about Successful School Leadership. London: National College of School Leadership.

OCDE. (2009). Informe TALIS: la creación de entornos eficaces de enseñanza y aprendizaje. Sintesis de los primeros resultados. En línea; disponiblke en: http://168.255.201.80/TALIS2009/ Sintesis_TALIS_Internacional.pdf (15/05/2012). 
Pedraja, L., Rodríguez-Ponce, E., Barreda, M., Sagredo, O. \& Segovia, C. (2009). Estilos de liderazgo y resultados del sistema de medición de la calidad de la educación: un estudio empírico en los colegios básicos de la ciudad de Arica-Chile. Ingeniare. Revista Chilena de Ingeniería, vol. 17, n. 1, 21-26.

Pont, B., Nusche, D. \& Moorman, H. (2009). Mejorar el liderazgo escolar. Política y Práctica (vol.1). En línea; disponible en: http://www.oecd.org/edu/school/44374937.pdf (15/05/2012).

Purkey, S. \&Smith, M. (1985).School reform: The district policy implications of the effective school literature. Elementary School Journal, n. 85, 353-389.

Ramos, I. (2005). Liderazgo didáctico. Cuadernos de Investigación en la educación, $n$. 20. En línea; disponible en: http://cie.uprrp.edu/cuaderno/ediciones/20/01.html (15/05/2012).

Reynolds, D. y Colaboradores (1997). Las escuelas eficaces: Claves para mejorar la enseñanza. Madrid: Santillana

Robinson, V., lloyd, C. \&Rowe, K. (2008). The Impact of Leadership on Student Outcomes: An Analysis of the Differential Effects of Leadership Types. Educational Administration Quarterly, vol. 44, n. 5, 635-674.

Scheerens, J. (1990). School effectivenes and the develoment of process indicator of school functioning. Effectiveness and School Improvement, vol. 1, n. 1, 371-403.

Scheerens, J. (1996) ¿Puede la base del conocimiento de la eficacia docente guiar la dirección de centros?. En Actas del segundo congreso internacional sobre dirección de centros docentes (pp. 779-805). Bilbao: ICE-Universidad de Deusto.

Sergiovanni, J.L. (1984). Leadership and excellence in scholling. Educational Leadership, n. 41, 4-13.

Thieme, P (2002). Liderazgo y Eficiencia en la Educación primaria. El caso de Chile. Tesis doctoral. Departamento de Economía de la Empresa. Barcelona: Universidad Autónoma de Barcelona.

Uribe, M (2005). El Liderazgo docente en la construcción de la cultura escolar de calidad. Un desafío de orden superior. PREAL, vol. 1, n. 1. 107-119.

Volante, P; Isla, P; Castro, P. \& Muller, M. (2002). Estándares para el Liderazgo Educativo. Boletín de Investigación Educacional, vol. 17., 101-118.

Volante, P. (2008). Influencia del liderazgo Instruccional en resultados de aprendizaje. En Maureira, O. (Ed.), Perspectivas de gestión para la innovación y el cambio educativo. Santiago: Universidad Católica Raúl Silva Henríquez. 
